\title{
MLD Model of Boiler-Turbine System Based on PWA Linearization Approach
}

\author{
M. Sarailoo*, B. Rezaie, Z. Rahmani \\ Intelligent Sy stem Research Group, Faculty of Electrical and Computer Engineering, Babol University of Technology,Babol, 47148-71167, \\ Iran
}

\begin{abstract}
In this paper we consider boiler-turb ine's nonlinear dynamics and linearize the nonlinear parts based on the piecewise affine method in order to obtain a mixed logical dynamical model of the system. By using piecewise affine approach for linearization of the system's nonlinear equations, the obtained linearized model switches between different modes based on its parameters, so acquired piece wise affine model can be categorized in the switching hybrid system class. We model the linearized boiler-turbine system in a mixed logical dynamical model of the hybrid systems using hybrid system's description language and hybrid toolbox. Mixed logical dynamical model describes system by two linear equ ations and one linear inequality with a reasonable accuracy and considering the constraints in the system. We provide a comparison between the acquired mixed logical dynamical model using piecewise affine linearization method and the actual boiler-turbine system through simulation and show the efficiency of the mixed logical dynamical model to describe the Boiler-Turbine system.
\end{abstract}

Keywords Boiler-Turbine System, Hybrid System, Piecewise Affine Method, Mixed Logical Dynamical Model, Modelling Nonlinear System

\section{Introduction}

In the study of power systems having a desirable model of system is important. A boiler-turbine system is an energy conversion system that consists of a steam boiler and a turbine which uses chemical energy to generate electricity. Boiler-turbine system model first was represented at 1972 using nonlinear equations based on the boiler-turbine plant P16/G16 at the Sydsvenska Kraft AB Plant in Malmo, Sweden[1]. A schematic diagram of the boiler-turbine unit is shown in Figure.1. After that the boiler-turbine's nonlinear model was studied widely in many articles and was undergone a number of amends to provide a better description of system[2,3]. In order to design a controller some researchers used nonlinear form of system's equations [5,6, 10$]$ and in other works the linear form of the system was used[4,7-9].

The boiler-turbine system can be modeled as a multi-input multi-output (MIMO) nonlinear system. This system is strongly coupled and is subject to various constraints on both inputs and outputs. In literatures that used the linearized form of boiler-turbine system, they obtained a linearized model using a truncated Tylor series expansion of nonlinear

* Corresponding author:

M.sarailoo@gmail.com (M. Sarailoo)

Published online at http://journal.sapub.org/control

Copyright (C 2012 Scientific \& Academic Publishing. All Rights Reserved equations around operating points [4,7-9]. Linearization around operating points provides a relatively accurate description about system behaviour around these points, however for using of this method we should have nominal states' and inputs' values at operating points which are not readily available.

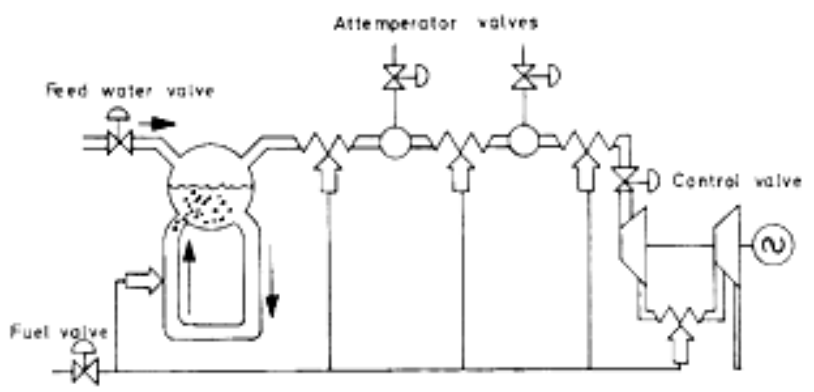

Figure 1. schematic diagram of the boiler-turbine unit

Because of improvement in computational methods and hardware, today the control's technology is moving toward intelligent and computer based controller methods namely model predictive control. For using such control methods, we need to provide a model of system which describes the system's behaviour properly. A good way for providing a proper model of different classes of systems is using hybrid system's modelling approach specially mixed logical dynamical (MLD) method which describes system using two linear equations and one linear inequality. In order to obtain a MLD model of a system first the system's equations need 
to be linearized, so we use piecewise affine (PWA) approach for linearization in which the space is divided into different pieces and in each section a linear equation is used for description of system behaviour in that section[11].

Here we use the boiler-turbine's nonlinear equations which represented in[3], to obtain a linear model of boiler-turbine system in the mixed logical dynamical fra me work using piece wise affine approach for linearization. The rest of the paper is as follows. In Section 2, we introduce the mathematical model of the boiler-turbine system and use piecewise affine method for linearization. Next in section 3, we acquire a MLD model and provide simulations and comparisons of the achieved MLD model and the actual system in identical condition. Finally, concluding remarks are drawn in Section 4.

\section{Mathematical and Linear Model of Boiler-Turbine System}

In this section, we consider the boiler-turbine system's continuous-time dynamical equations as follow[3]:

$$
\begin{gathered}
\frac{d p}{d t}=-0.0018 u_{2} p^{9 / 8}+0.9 u_{1}-0.15 u_{3} \\
\frac{d p 0}{d t}=\left(0.073 u_{2}-0.016\right) p^{9 / 8}+0.1 p 0 \\
\frac{d p f}{d t}=\frac{\left(141 u_{3}-\left(1.1 u_{2}-0.19\right) p\right)}{85}
\end{gathered}
$$

where $p, p_{0}$ and $p_{f}$ are drum pressure $\left(\mathrm{kg}_{\mathrm{g}} \mathrm{cm}^{2}\right)$, power output (MW) and fluid density $\left(\mathrm{kg}^{\mathrm{c}} \mathrm{cm}^{3}\right)$, respectively. The inputs to the system are $\mathrm{u}_{1}, \mathrm{u}_{2}$ and $\mathrm{u}_{3}$ which respectively indicate to fuel flow valve position, steam control valve position and feedwater flow valve position, and have a value in the interval $[0,1]$. Changes in valves' position have the following limitations:

$$
\begin{gathered}
\left|\frac{d u_{1}}{d t}\right| \leq 0.007 / \mathrm{sec} \\
-2 / \mathrm{sec} \leq \frac{d u_{2}}{d t} \leq 0.007 / \mathrm{sec} \\
\left|\frac{d u_{3}}{d t}\right| \leq 0.05 / \mathrm{sec}
\end{gathered}
$$

The output to the system are $\mathrm{p}, \mathrm{p} 0$, and $\mathrm{Xw}$ which are respectively drum pressure, output power, and drum water level (in meters). Two first output are system's states and readily available, whereas water level is found through the following relationships:

$$
\begin{gathered}
X_{w}=0.05\left(0.13073 p_{f}+100 \alpha_{c s}+\frac{q_{e}}{9}-67.975\right) \\
\alpha_{c S}=\frac{\left(1-0.001538 p_{f}\right)(0.8 p-25.6)}{p_{f}(1.0394-0.0012304 p)} \\
q_{e}=\left(0.854 u_{2}-0.147\right) p+45.59 u_{1}-2.514 u_{3}-2.096
\end{gathered}
$$

where $q_{e}$ is the evaporation rate $(\mathrm{kg} / \mathrm{sec})$ and $\alpha_{c s}$ is the steam quality.

For obtaining the MLD model of the boiler-turb ine system according equations (1), (3) and by using Hybrid Systems Description Language (HYSDEL), first the nonlinear terms in these equations must be omitted. These nonlinear terms are presented at equation (4).

$$
\begin{aligned}
& f_{1}\left(p, u_{2}\right)=u_{2} p^{9 / 8}, \quad f_{2}\left(p, u_{2}\right)=u_{2} p, \\
& f_{3}\left(p, p_{f}\right)=\frac{\left(1-0.001538 p_{f}\right)(0.8 p-25.6)}{p_{f}(1.0394-0.0012304 p)} \text { and } f_{4}(p)=p^{9 / 8}
\end{aligned}
$$

As we mentioned earlier, for linearization we use the PWA approach[11]. So we considered that the $p, p_{f}$, and $u_{2}$ respectively have a value in the interval $[70,145],[200,600]$, and $[0,1]$ which describe operating space of boiler-turbine system, and divide each space into the 4 equal separate sections. In consequence, the nonlinear terms $f_{1}, f_{2}$, and $f_{3}$ consist 16 sections and $f_{4}$ consists 4 sections. Now we describe the nonlinear terms in each section with a linear equation. For $f_{1}, f_{2}$, and $f_{3}$ we represent nonlinear terms using surfaces as follow:

$$
z=a_{i} x+b_{i} y+c_{i} \quad i=1, \ldots 16
$$

and in order to describe $f_{4}$ we use linear lines with following equations:

$$
y=\hat{a}_{i} x+\hat{b}_{i} \quad i=1, \ldots, 4
$$

where $a_{i}, b_{i}, \hat{a}_{i}$ and $\hat{b}_{i}$ are constant factors related to the $i$-th section.

For examp le accord ing to equation (5-1) the surface which describes the nonlinear terms $f_{3}$ in the interval $p_{f}=[300,400]$ and $p=[107.5,126.3]$ is as follow:

$$
f_{3}=(-0.0006 .327984) p_{f}+(0.0013753) p+(0.16542755)
$$

similarly, we can obtain the linear form of equations (4) in each section. Comparisons between nonlinear and linear form of $f_{1}, f_{2}, f_{3}$, and $f_{4}$ are shown in Figure.2.

Now according to linearized form of nonlinear terms, the boiler-turbine system's dynamics can be written in the PWA configuration which Equation (6) shows general formu lation of that[11].

$$
\begin{aligned}
& x_{k+1}=A_{i} x_{k}+B_{i} u_{k}+h_{i} \\
& y_{k}=C_{i} x_{k}+D_{i} u_{k}+g_{i}
\end{aligned} \quad \text { if } \quad \Omega_{i}=\text { true }
$$

where, $k$ is the discrete-time step. $x, u$, and $y$ denote states, inputs, and outputs, respectively. $\Omega_{i}$ indicates a set of conditions which defines $i$-th section of space (for boiler-turbine we have 64 section). $A_{i}, B_{i}, h_{i}, C_{i}, D_{i}$, and $\mathrm{g}_{\mathrm{i}}$ are proper time-invariant matrix related to section $i$. The concept of PWA model is depicted in Figure.3.

In next section according to the linear discrete-time equations of boiler-turbine system, which were obtained in this section, and using HYSDEL we will acquire the MLD model of system. 

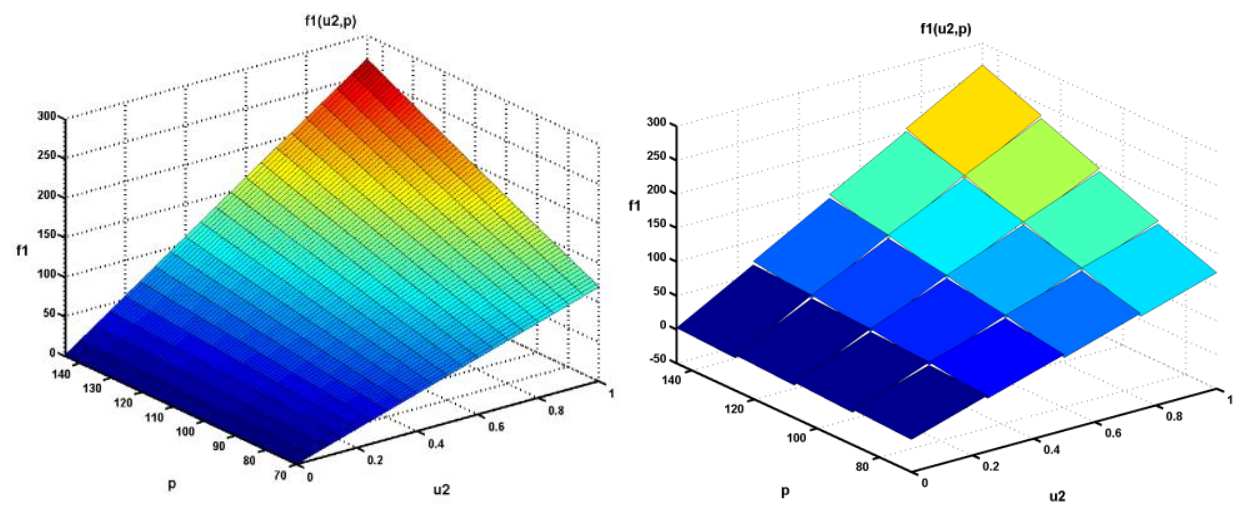

a)
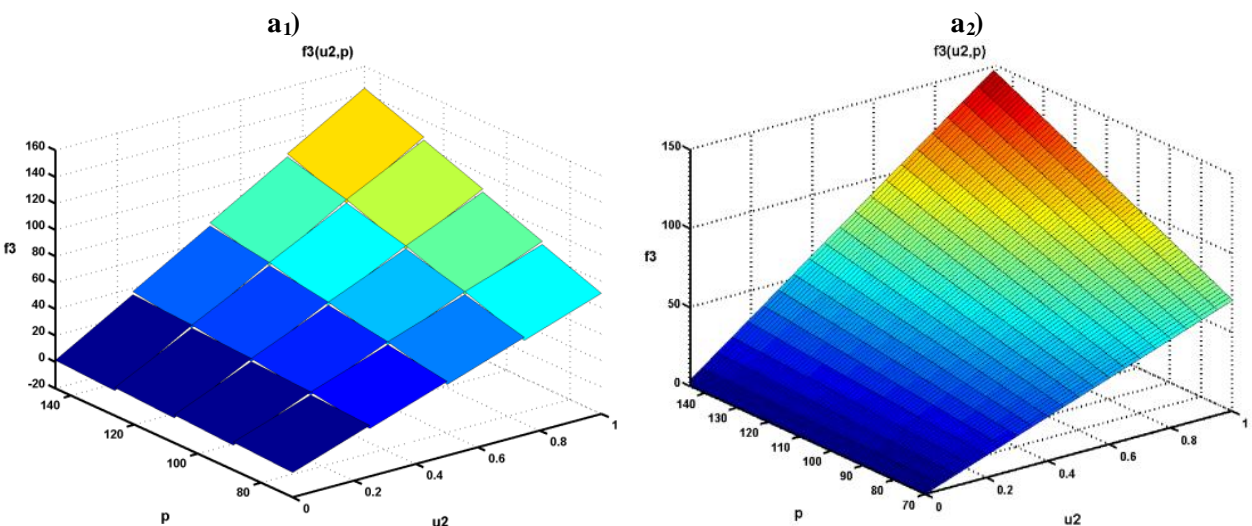

b1)

b2)
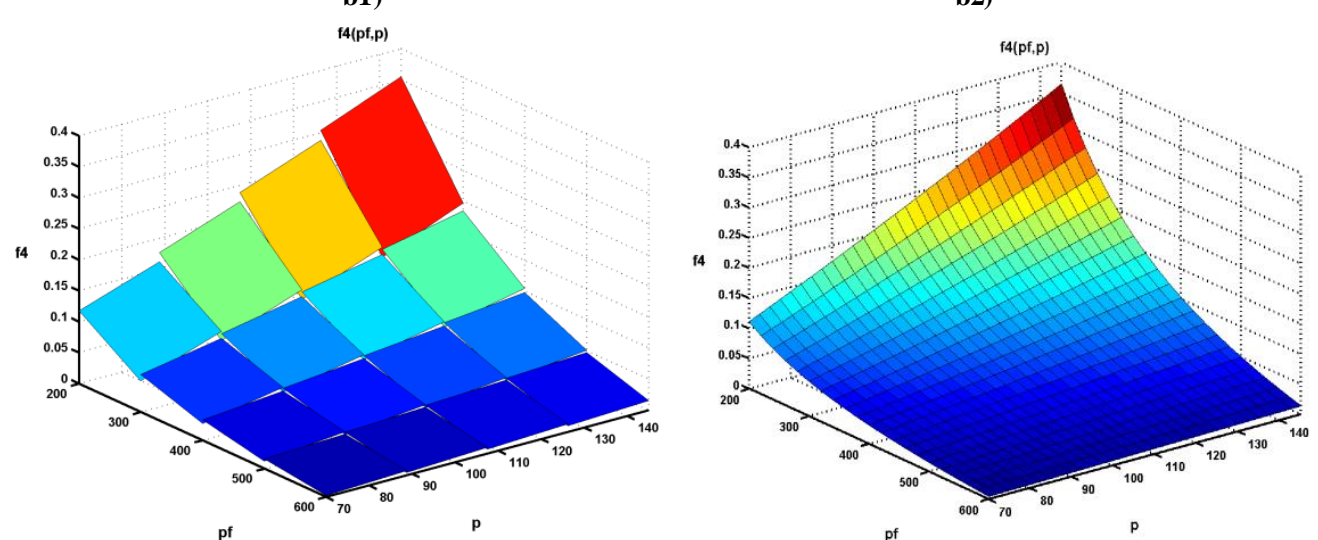

c1)

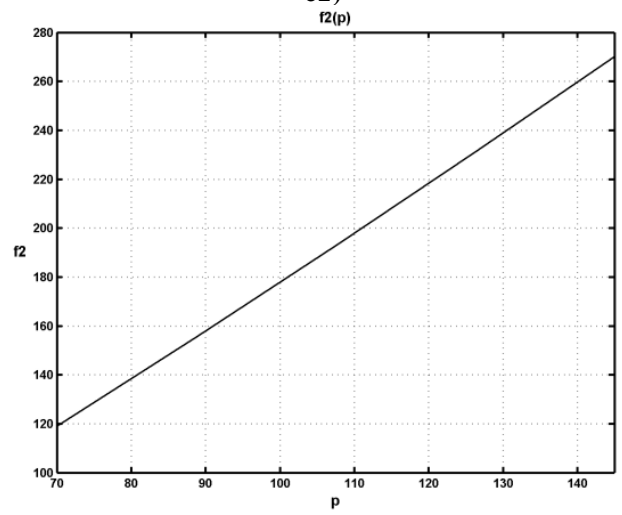

c2)

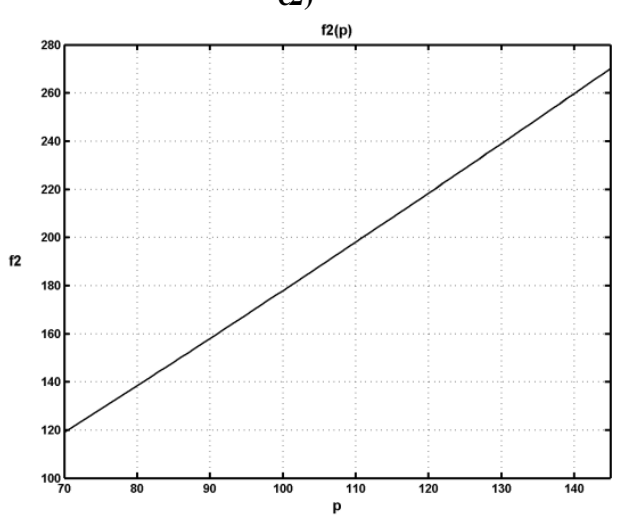

d1)

d2)

Figure 2. comparison between nonlinear and linear form of $f 1$ (a), $f 2$ (b), $f 3$ (c), and $f 4$ (d): left pict ures are showed linearized form and right pict ures are showed nonlinear form 


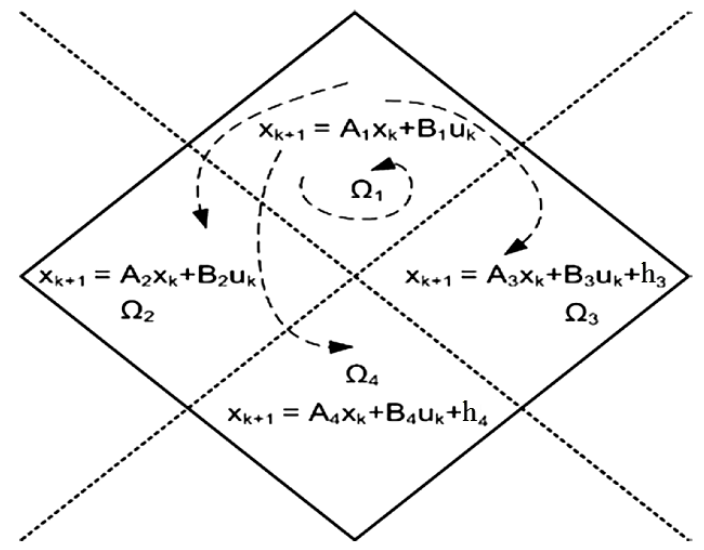

Figure 3. a bounded PWA model: in each section linear dynamics describe system behaviour

\section{Mixed Logical Dynamical Model and Simulation}

There are many different methods for modelling of hybrid system, namely Piecewise affine, Mixed logical dynamical, Linear complementarity, and Max-min-plus-scaling[12].
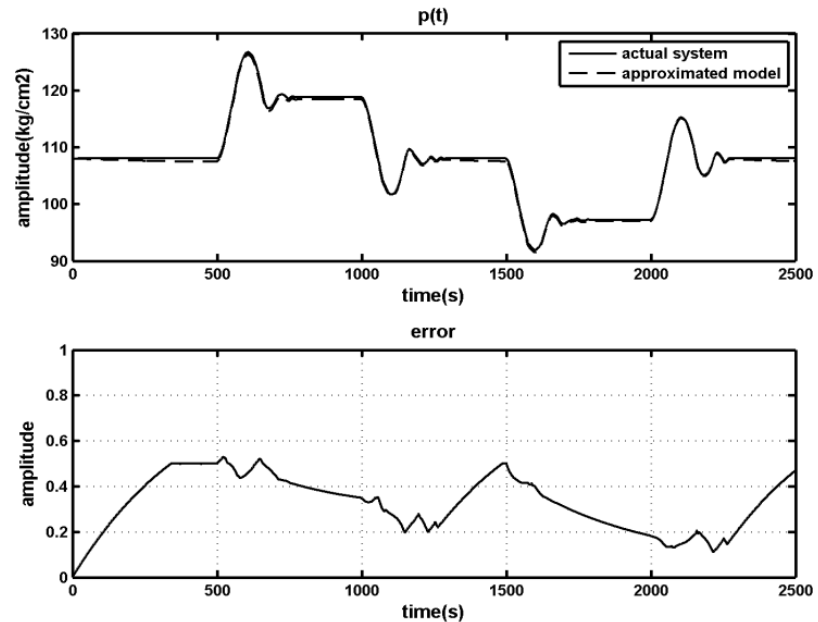

a)
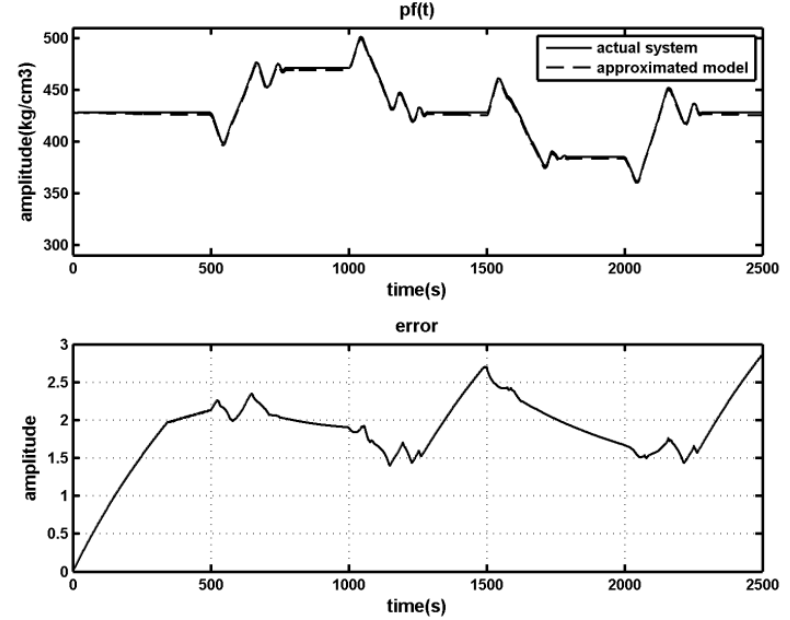

c)
Among these models, MLD method is more common and has less complexity than other methods which models hybrid system using two linear equations (7-1) and (7-2) and one linear inequality (7-3). MLD approach has the following structure[13]:

$$
\begin{gathered}
x(k+1)=A x(k)+B_{1} u(k)+B_{2} \delta(k)+B_{3} z(k) \\
y(k)=C x(k)+D_{1} u(k)+D_{2} \delta(k)+D_{3} z(k) \\
E_{2} \delta(k)+E_{3} z(k) \leq E_{1} u(k)+E_{4} x(k)+E_{5}
\end{gathered}
$$

where $x=\left[\begin{array}{ll}x_{c} & x_{l}\end{array}\right]^{T}, x_{c} \in R^{n_{c}, x_{l} \in\{0,1\}^{n} l}$ is the system state, $\left.\quad y=\left[\begin{array}{ll}y_{c} & y_{l}\end{array}\right]^{T}, y_{c} \in R^{p_{c}, y_{l} \in\{0,1\}}\right\}_{l} \quad$ and

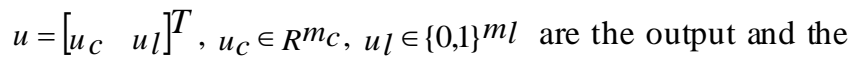
input signal, respectively. $\delta \in\{0,1\}^{r_{l}}$ denotes the logical auxiliary variables, and $z \in R^{r c}$ denotes the continuous auxiliary variables. The indexes $c$ and $l$ represent the type of the variables and respectively indicate continuous and logical. $A, B_{i}, C, D_{i}$ and $E_{i}$ are proper and time-invariant matrixes.
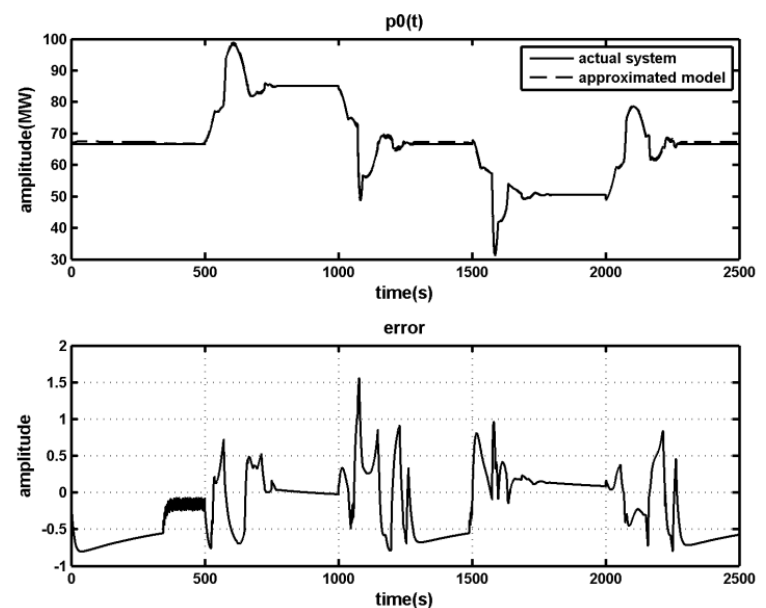

b)
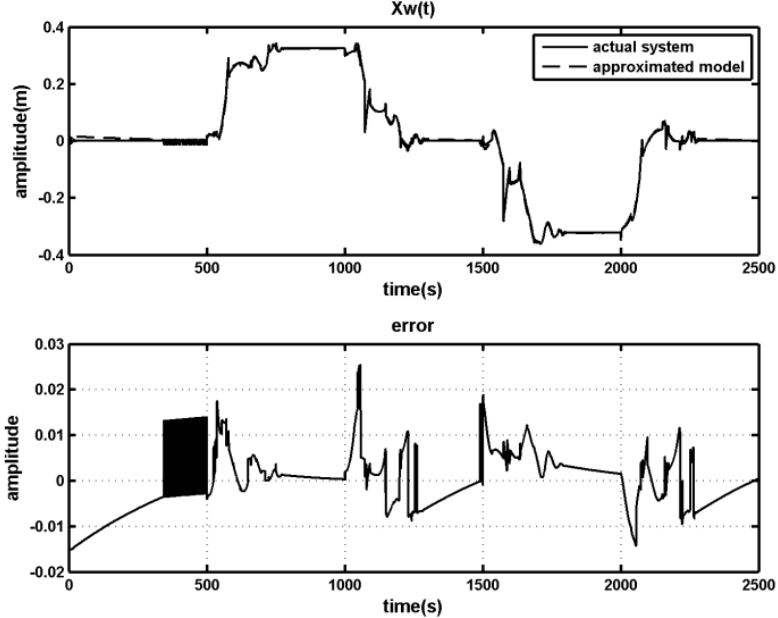

d)

Figure 4. actual system's states and outputs (solid-line) in comparison of MLD model's states and outputs (dashed-line) under identical conditions and inputs' signals: a)drum pressure, b) power output, c)fluid density, and d)drum water level 
Before obtaining the MLD model of boiler-turbine system based on the PWA model (equation (6)), in order to impose the inputs' constraints that were defined at equations (2); we introduce 3 new states (8-1) and 3 new equations (8-2) as follow:

$$
\begin{gathered}
p u_{i}(k+T s)=u_{i}(k) \quad i=1,2,3 \\
d u_{i}(k)=\frac{u_{i}(k)-p u_{i}(k)}{T s} \quad i=1,2,3
\end{gathered}
$$

where $T_{s}$ is sampling time. ${ }^{p u}$ and $d u$ denote the new state variable and input's pace, respectively.

Now the MLD model (7) of boiler-turbine system is obtained by using HYSDEL and hybrid toolbox $[14,15]$. The MLD model of the system by using PWA method for linearization has the following properties:

1. Sampling time $\left(T_{s}\right)$ is $1 \mathrm{~s}$.

2. 6 states ( 6 continuous: -0 binary), 3 inputs ( 3 continuous: -0 binary), 3 outputs ( 3 continuous: -0 binary).

3. 63 continuous auxiliary variables, 45 binary au xiliary variables, 392mixed-integer linear inequalities.

In following, we provide a comparison between approximated MLD model and actual system under identical conditions and inputs signals. The results of simulation are shown in Figure.4.

By looking at Fig.4, it is obvious that using piecewise affine approach for linearization describes actual system behavior at a reasonable accuracy level. In order to increase the accuracy of the MLD model of boiler-turbine system we should increase the number of partitions of the piecewise affine approach. However according to the MLD models' properties, we should consider that increase in partitions' number causes increase in the nu mber of au xiliary variables and mixed-integer linear inequalities related to the MLD model. Consequently, we need more computational effort to use this MLD model in order to design a controller for the actual boiler-turbine system

\section{Conclusions}

As we showed in this paper hybrid systems modelling approaches can be used not only for systems with inherently hybrid behaviours but also nonlinear systems, by using local linearization method namely PWA. We showed using MLD model method for modelling boiler-turbine system as a hybrid system makes us able to impose constraint directly into the system model and provides a very simple, systematic, and relatively accurate model of system. This accuracy comes from the PWA method which we used for linearization of nonlinear terms but increase in accuracy also causes increase in computational effort for designing a proper controller based on the MLD model. So we should make a balance between accuracy and computational cost according to our purposes and available equip ments.

\section{REFERENCES}

[1] K.J. Astrom and K. Eklund, "A simplified non-linear model of a drum-boiler-turbine unit," International Journal of Control, vol. 16, pp. 146-169, 1972.

[2] A.J. Morton and P.H. Price, "The controllability of steam output, pressure and water level in drum boilers," Steam at Work: Industrial and Marine Steam Plant, pp. 75-84,1977.

[3] R.D. Bell and KJ. Astram, "Dynamic models for boiler-turbine alternator units: Data logs and parameter estimation for a 160 MW unit," Report TFRT-3192, Lund Institute of Technology, Sweden, 1987.

[4] R. Dimeo and K.Y. Lee, "Boiler-turbine control system design using a genetic algorithm," IEEE Trans. Energy Conversion, vol. 10, pp. 752-759, 1995.

[5] A.B Abdennour and K.Y. Lee, "An Autonomous Control System For Boiler-Turbine Units," IEEE Trans. Energy Conversion, vol. 11, pp. 401-406, 1996.

[6] U.C Moon and K.Y. Lee, "A boiler-turbine system control using a fuzzy auto-regressive moving average (FARMA) model," IEEE Trans. Energy Conversion, vol 18, pp. 142-148, 2003.

[7] P.Ch Chen and J.S. Shamma, "Gain-scheduled $\ell$ 1-optimal control for boiler-turbine dy namics with actuator saturation," Journal of Process Control, vol. 14, pp. 263-277, 2004.

[8] W. Tan, H.J. Marquez, T. Chen and J. Liu, "Analysis and control of a nonlinear boiler-turbine unit," Journal of Process Control, vol. 15, pp. 883-891, 2005.

[9] J. Wu, M. Kurg, S.K. Nguang, J. Shen and Y.G. Li, "Hळ fuzzy tracking control for boiler-turbine systems," IEEE Int. Conf. Control and Automation, Christchurch, New Zealand, pp.1980-1985, 2009.

[10] J. Wu, J. Shen, M. Krug, S.K. Ngu ang and Y. Li, "GA-based nonlinear predictive switching control for a boiler-turbine system," Journal of Control Theory and Applications, vol. 10, pp. 100-106, 2012.

[11] E. Sontag, "Nonlinear regulation-The piecewise linear approach," IEEE Trans. on Automatic Control, vol. 26, pp. 346-358, 1981.

[12] W.P.M.H. Heemels, B. De Schutter, A. Bemporad, "Equivalence of hybrid dynamical models," Automatica, vol. 37, pp. 1085-1091, 2001.

[13] A. Bemporad, M. Morari, "Control of systems integrating logic, dynamics, and constraints," Automatica, vol. 35, pp. 407-427, 1999.

[14] F.D. Torrisi, A. Bemporad, "HYSDEL-a tool for generating computational hybrid models for analysis and synthesis problems," IEEE Trans. on Control Syst. Technol., vol. 12, pp. 235-249, 2004.

[15] A. Bempor, “ Hybrid Toolbox v.1.2.6,” http://control.ee.ethz .ch/ hybrid 